\title{
Direct T2 quantification to detect myocardial edema in patients with myocarditis and tako-tsubo cardiomyopathy
}

\author{
Paaladinesh Thavendiranathan*, Michael Walls, Shivraman Giri, David Verhaert, Orlando P Simonetti, \\ Subha V Raman
}

From 2011 SCMR/Euro CMR Joint Scientific Sessions

Nice, France. 3-6 February 2011

\section{Background/objective}

T2-weighted cardiovascular magnetic resonance (T2W-CMR) has shown utility in diseases such as myocarditis and tako-tsubo cardiomyopathy (TTCM) where myocardial edema may be the predominant pathological abnormality. Since these conditions may affect many myocardial regions, a more quantitative approach may help identify extent of myocardial involvement and reduce uncertainty in qualitative interpretation of T2WCMR. We sought to evaluate the diagnostic utility of quantitative $\mathrm{T} 2$ mapping in patients presenting with acute myocarditis or TTCM.

\section{Methods}

Consecutive patients referred for CMR assessment of myocarditis or TTCM who met established diagnostic criteria were enrolled. Healthy volunteers served as controls. Patients' CMR studies included T2 mapping [1] on the identical 1.5T scanner with a 12-element phasedarray coil (MAGNETOM Avanto). One reviewer blindly scored each patient's cine and late gadolinium enhancement (LGE) images across 17 myocardial segments. Regions with wall motion abnormality, LGE-positivity and/or visually-apparent T2 map abnormality defined presence of myocardial involvement. Myocardial T2 values were obtained by encircling a region of at least 15 pixels (Figure 1). In controls, T2 values were measured across an entire mid-short axis slice. Abnormal $\mathrm{T} 2$ values were defined as exceeding $2 \mathrm{SDs}$ above the mean $\mathrm{T} 2$ of controls.

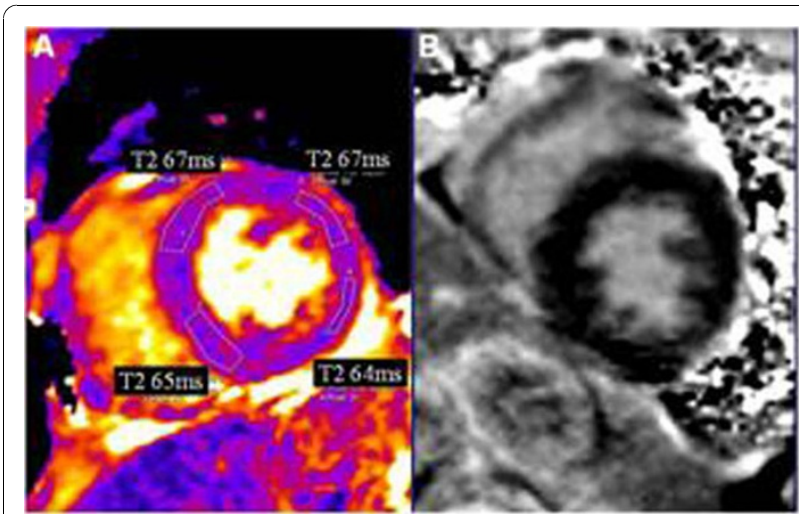

Figure 1 (A) Basal short axis T2 map shows elevated T2 in a patient with myocarditis involving most of the basal segments. (B) Corresponding LGE image shows no hyperenhancement.

\section{Results}

Fourteen patients (10 with myocarditis and 4 with TTCM) age $43 \pm 17$ years and 14 controls were enrolled. Patients' peak troponin was $13 \pm 12 \mathrm{ng} / \mathrm{ml}$, time from admission to CMR $1.9 \pm 2.3$ days, and LVEF $47 \pm 9 \%$. T2 maps were successfully obtained in all patients. Patients' T2 in involved myocardial segments was $66.1 \pm 4.0 \mathrm{~ms}$ vs. $54.9 \pm 2.5 \mathrm{~ms}$ in healthy volunteers' myocardium $(\mathrm{p}<0.001)$. $\mathrm{T} 2$ values were similarly elevated in patients with myocarditis and TTCM $(65.3 \pm 3.4$ and $69.0 \pm 5.1 \mathrm{~ms}$, respectively; $\mathrm{p}=0.3$ ). No TTCM had abnormal LGE (Figure 2); however, 7/10 patients with myocarditis showed classic LGE abnormalities. Among patients with abnormal LGE, 5 had abnormal T2 values involving $5 \pm 2.5$ segments beyond the LGE-positive areas (Figure 3 ).

\footnotetext{
The Ohio State University, Columbus, OH, USA
} 


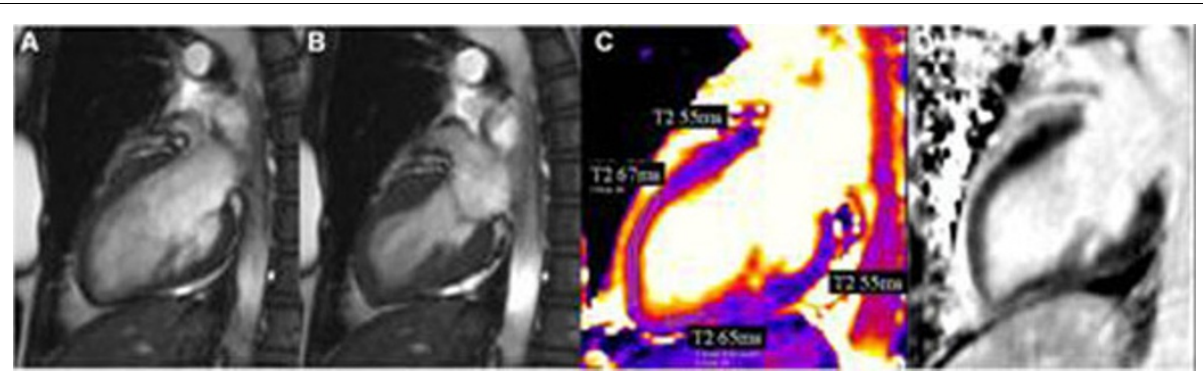

Figure 2 Vertical long axis diastolic (A) and systolic (B) SSFP cinc frames illustrate apical ballooning. (C) T2 map in the same view shows elevated T2 in the apical LV segments and (D) LGE image shows no hyperenhancement.

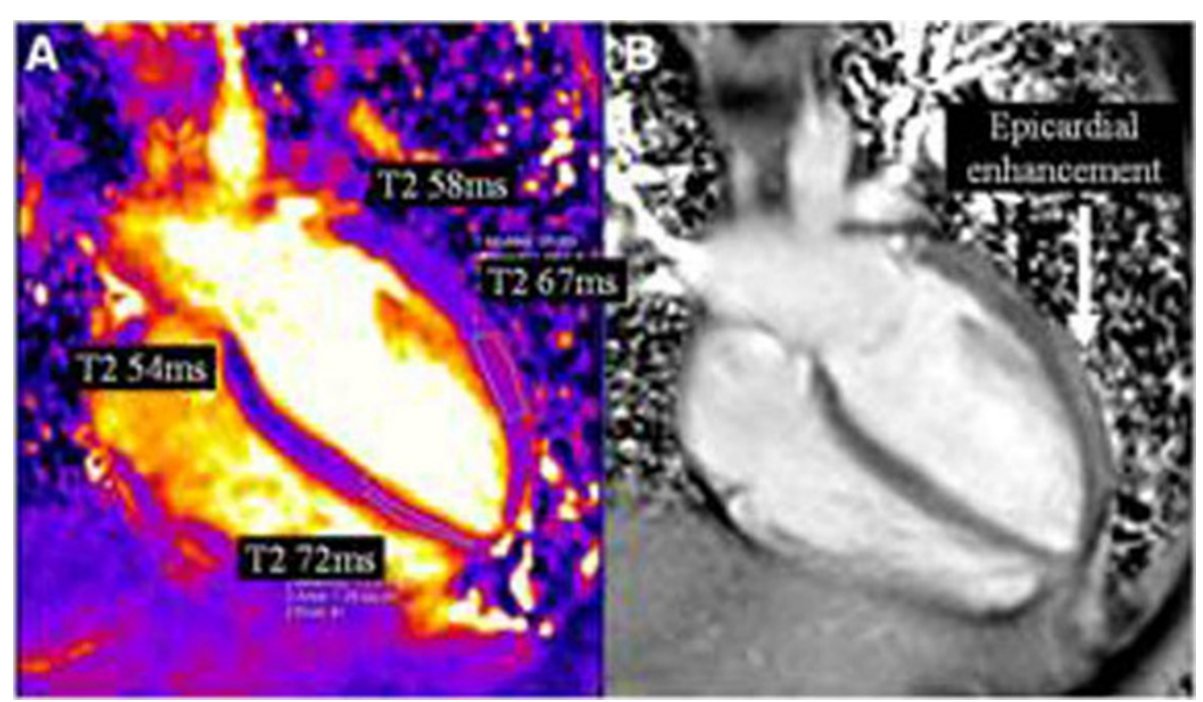

Figure 3 (A) T2 map in a horizontal long axis view in a patient with myocarditis illustrates high T2 values both in the distal septum and lateral wall. (B) Corresponding LGE imaging shows epicardial hyperenhancement online in the lateral wall.

\section{Conclusion}

T2 quantification identifies myocardial abnormalities in patients with myocarditis and TTCM. Abnormal T2 occurred in the absence of LGE-positivity in all TTCM and $30 \%$ of myocarditis patients. In addition, T2 mapping identified areas of edema beyond LGE-positive regions in myocarditis. T2 mapping may provide additional information beyond cine and LGE, suggesting a more comprehensive assessment of myocardial involvement in both myocarditis and TTCM.

Published: 2 February 2011

\section{Reference}

1. J Cardiovasc Magn Reson 2009, 11:56.

\section{doi:10.1186/1532-429X-13-S1-P313}

Cite this article as: Thavendiranathan et al: Direct T2 quantification to detect myocardial edema in patients with myocarditis and tako-tsubo cardiomyopathy. Journal of Cardiovascular Magnetic Resonance 201113 (Suppl 1):P313.

\section{Submit your next manuscript to BioMed Central} and take full advantage of:

- Convenient online submission

- Thorough peer review

- No space constraints or color figure charges

- Immediate publication on acceptance

- Inclusion in PubMed, CAS, Scopus and Google Scholar

- Research which is freely available for redistribution 